\title{
8 OBERSTE KOMMANDOBEHÖRDEN UND DIENSTSTELLEN DER WEHRMACHT
}

Militärisches Schriftgut, das sich bei Kriegsende in Gebieten befand, die von der Roten Armee erobert oder besetzt wurden, wird weiterhin in Moskau verwahrt; nähere Angaben darüber, wie hoch darin der Anteil von Akten zentraler Kommandobehörden und von regional zuständigen Dienststellen ist, sind nicht möglich, doch dürfte es sich vorwiegend um Kriegstagebücher von Truppenteilen handeln. Die nachstehenden Bestandsbeschreibungen berücksichtigen überwiegend die direkt oder über das ehemalige Militärarchiv der DDR in das BA-MA gelangte Überlieferung, bei der Schriftgut der Kriegsmarine bis auf Aktenreste des Marineoberbauamts Berlin fehlt.

Lit.: B. WEGENER: Deutsche Aktenbestände im Moskauer Zentralen Staatsarchiv. 1992. S. 314 ff. - G. ALY, S. HEIM: Das Zentrale Staatsarchiv in Moskau. 1992. S. 20.

\subsection{Oberste Kommandobehörden mit nachgeordneten zentralen Dienststellen}

Bei den in Moskau (ZDM, Best. 1280, 50 Bde) nachgewiesenen Akten aus dem Oberkommando der Wehrmacht handelt es sich ebenso um zufällige Reste wie bei den einzelnen Bänden im BA DH, zu denen ein Bericht der Abwehrabteilung des Reichswehrministeriums über die Spionageaffäre Sosnowski, Unterlagen über Kriegsberichterstatter und eine Liste der Ic-Offiziere bei Heeresgruppen, Armeen und Korps (1944) aus dem Oberkommando des Heeres gehören.

\subsubsection{Kriegsgeschichtliche Forschungsanstalt des Heeres, Potsdam} BA-MA, Best. W 10; ZDM MO, Best. 545

Hauptaufgabe der 1935 aus der Historischen Abteilung des Reichsarchivs hervorgegangenen und zuletzt dem Beauftragten des Führers für die militärische Geschichtsschreibung (Aktenrest BA-MA, Best. RW 9) unterstellten Behörde war der AbschluB der Bearbeitung der amtlichen Darstellung der Geschichte des Ersten Weltkriegs und der Kämpfe deutscher Truppen und Freikorps im AnschluB daran. Der BA-Bestand (2353 Bde, 50 Kts unverzeichnete Unterlagen, vor 1914-1945) enthält Akten über Organisation und Dienstbetrieb (102 Bde, 1914-1944), u. a. über Trennung von Reichs- und Heeresarchiv, Entstehung und Fortführung des Weltkriegswerks, Schriftwechsel des Chefs mit führenden Offizieren (5 Bde, 1925-1945) vor allem aber Materialsammlungen zu den Publikationen mit unveröffentlichten Manuskripten, Tagebüchern und Erfahrungsberichten; sie sollten auch einem Vergleich zwischen der Kriegführung in beiden Weltkriegen dienen, z. B. durch eine Untersuchung „Verdun 1916 und 1940“ mit den Ergebnissen einer "Schlachtfelderfahrt". Ergänzungsüberlieferung enthält der Nachlaß des Präsidenten Wolfgang Foerster (BA-MA, Best. N 121, 44 Bde, 1867-1960). Zum Moskauer Bestand (465 Bde) dürften vorwiegend Unterlagen über Kämpfe an der Ostfront und im Baltikum gehören.

Lit.: R. BRÜHL: Militärgeschichte und Kriegspolitik. 1973. - H. OTTO: Der Bestand Kriegsgeschichtliche Forschungsanstalt des Heeres. 1992. 


\subsubsection{Chef der Heeresarchive}

BA-MA, Best. RH 18; ZDM MO, Best. 1256, 1275

Aus dem Büro des Amtschefs General der Artillerie Friedrich v. Rabenau sind nur wenige Akten überliefert, u. a. über Bildung, Aktenübernahmen, Leitung und Organisation der Heeresarchive (4 Bde, 1940-1942) und das österreichische Kriegsarchiv in Wien (1936-1938), Teile des Kriegstagebuchs, eine Liste der Beauftragten in den besetzten Gebieten (1939-1940), Verzeichnisse zu Sammlungen des Beauftragten in Belgien und Nordfrankreich (3 Bde, 1940-1944) und von der Abwicklungsstelle des Beauftragten in den Niederlanden übersandten Akten (1940). Sie werden ergänzt durch den Nachlaß Rabenaus (BA-MA, Best. N 62).

Den Hauptteil der Überlieferung bilden Akten des Heeresarchivs Potsdam, die beim Luftangriff 1945 nicht vernichtet wurden; ein Teil davon befand sich schon früher im BA-MA, ein anderer kam aus dem ehemaligen Militärarchiv der DDR hinzu (379 Bde, 1920-1945), ein weiterer liegt noch in Moskau (108 und 163 Bde). Sie besteht aus Unterlagen über organisatorische und Benutzungsangelegenheiten, archivfachliche Fragen, Bestandsübersichten, Formations-, Nachlaß-, Bücher- und Aktenverzeichnissen und enthält auch Akten der Beauftragten des Chefs der Heeresarchive in Frankreich, Belgien, den Niederlanden und der Aktensammelstellen West und Süd, darunter der Schlußbericht über den Einsatz der Gruppe Archivwesen beim Militärbefehlshaber Frankreich (1940-1944), in Moskau (Best. 1387) wohl auch von der HeeresarchivZweigstelle Danzig-Oliva.

Lit.: F.-C. STAHL: Die Organisation des Heeresarchivwesens in Deutschland 1936-1945. 1977. - U. LÖBEL: Neue Forschungsmöglichkeiten zur preuBisch-deutschen Heeresgeschichte. 1992.

\subsubsection{E Reichsministerium der Luftfahrt und Oberkommando der Luftwaffe
ZDM MO, Best. 702}

Den Hauptteil des kleinen Bestandes (36 Bde, 1930-1945) bilden Berichte der Luftwaffenattachés in Rom, Paris, London, Tokio, Washington, Belgrad und Prag (22 Bde, 19331940) u. a. über den Spanischen Bürgerkrieg, ausländische Luftfahrtindustrie (mit Fotografien). Im übrigen handelt es sich um Druckschriften (5 Bde) und einzelne Vorgänge u. a. über Arbeitseinsatz, Luftschutzmaßnahmen, Meßstellen für Luftschadstoffe und eine Liste zwischen Februar und April 1945 gefallener Offiziere und Soldaten.

\subsection{Oberkommandos und Generalkommandos}

Berücksichtigt sind wie in Teil 1 nur Kriegstagebücher (KTB, vgl. dazu dort S. 441) und Akten von Stäben, die im Frieden und bei den Endkämpfen im Reichsgebiet 1944/45 entstanden sind.

\subsubsection{Oberkommandos von Heeresgruppen}

BA-MA, Best. RH 19; AdR, Best.07R136/1

Vom Gruppenkommando 3, Dresden, sind vor allem (insgesamt 36 Bde, 1937-1939) Akten über die Besetzung des Sudetenlandes und von Böhmen und Mähren vorhanden (25 Bde mit KTB, 1938-1938), außerdem über Manöver 1937 und den Einmarsch in Österreich. Vom dort am 1. April 1938 gebildeten Gruppenkommando 
5, Wien, sind Tages- und Stabsbefehle (1938-1939) und Unterlagen (AdR, insgesamt $34 \mathrm{Kts}$ ) aus folgenden Sachgebieten überliefert: Erfahrungen beim Einmarsch; Durchführung der Eingliederung des österreichischen Bundesheeres; Organisation und Dienstbetrieb (Geschäftsverteilungsplan 1939 auch BA-MA); Personalangelegenheiten der Offiziere (mit Beurteilungen, Abstammungsnachweisen), u. a. Verabschiedungen, Pensionskürzungen); Erhebungen über wegen nationalsozialistischer Betätigung gemaßregelte Angehörige des Bundesheeres und Wiedergutmachung dafür.

Von den 1944/45 an der Ostfront zuständigen Heeresgruppen hat nur das Kriegstagebuch mit Anlagen der von Himmler geführten Heeresgruppe Weichsel (Best. RH $19 \mathrm{XV}, 28$ Bde, davon 2 vom Chef des Stabes, 19 vom Ia, 2 vom Ic) als Quelle für die Kämpfe in Pommern und an der Oder größere Bedeutung; überliefertes Schriftgut der Heeresgruppen Nord (Ostpreußen), Ostmark und Mitte (Lausitz, Böhmen und Mähren) reicht nur selten über die Mitte 1944 hinaus.

Lit.: GUIDES to German Records, vol. 40 und 52.

\subsubsection{Oberkommandos von Armeen}

BA-MA, Best. RH 20

Kriegstagebücher und Akten sind in der Regel nur bis Mitte 1944 überliefert, darüber hinaus nur in Resten u. a. vom Panzer-AOK 3 (Pommern), AOK 4 (Ostpreußen), Panzer-AOK 4 (Niederschlesien), AOK 9 (Brandenburg), AOK 17 (Schlesien) und AOK 21 (Mecklenburg). Daß sich Unterlagen aus den Endkämpfen noch im Moskauer Bestand befinden, ist nicht auszuschließen. $\mathrm{Zu}$ verweisen ist darauf, daß auch Kriegstagebücher unterstellter Generalkommandos von Armee- und Panzerkorps und - seltener - von Divisionen bis 1945 reichen können (BA-MA, Best. RH 24, RH 26 - 29).

Lit.: GUIDES to German Records, vol. 42, 43, 47, 48, 53, 58.

\subsubsection{Chefs der Zivilverwaltung im Operationsgebiet}

Während sich Aktenreste des bis zum Aufbau der Zivilverwaltung 1939 für Oberschlesien zuständigen Militärverwaltungschefs mit Ausnahme von einigen Verordnungen und Berichten zu kulturellen Angelegenheiten aus dem Grenzschutz-Abschnittskommando 3 (KA, 2 Bde, 1939) als Vorakten im Schriftgut der Regierung Kattowitz befinden, sind folgende Bestände gesondert überliefert:

Chef der Zivilverwaltung beim Oberbefehlshaber der 8. Armee im Militärbezirk Posen POS, Best. 298

Vorhanden sind (93 Bde, 1939) u. a. Berichte über die Lage in den Kreisen mit Angaben über Kriegsschäden, Plünderungen und den volksdeutschen Selbstschutz (27 Bde), Stabs- und Sonderbefehle, Berichte (5 Bde), u. a. über Beschlagnahme von Geld und Wertgegenständen, Tagesmeldungen von Einsatzgruppen der Ordnungs- und Sicherheitspolizei, Dienstanweisungen, Verordnungen, Aufrufe an die Bevölkerung (5 Bde), Akten über Personalangelegenheiten (4 Bde), Organisation und Dienststellenverwaltung mit Sitzungsprotokollen (14 Bde), Jagd und Fischerei (2 Bde), Straßenbezeichnungen (2 Bde), Sicherstellung von Akten und Gebäuden (3 Bde), persönliche Angelegenheiten Greisers, Verkehr mit Verbindungsoffizieren, Polizei und Kommunalbehörden (3 Bde), Polizeiangelegenheiten (14 Bde, 1939-1940), darunter Unterlagen über polnische Beamte, deutsche Orte, Dienstpläne, Dolmetscher, Deutsche Volksliste. Ein kleiner Teilbestand ist mit Akten des Wehrkreiskommandos XXI überliefert (BA-MA, Best. RH 
53, 7 Bde), dabei ebenfalls Lageberichte und Unterlagen über Verwaltungsaufbau und Errichtung eines Sondergerichts.

Vom Posener Bestand wurden verfilmt (BA P, Best. PL-094, 4 Filme von 54 Bden) Berichte der Landräte in Kosten, Lissa, Ostrowo, Schroda, Schubin, Wirsitz, Wongrowitz, Gnesen, Hohensalza, Jarotschin, Kempen, Krotoschin, Mogilno, Neutomischel, Obornik, Samter, Turek, Wollstein, Wreschen, Pnin (Dietfurt), Birnbaum, der Oberbürgermeister von Hohensalza und Posen und des Polizeipräsidenten Posen, Protokolle über Besprechungen über Polizei und die Einrichtung eines KL, Stabs- und Tagesbefehle, u. a. über Aufgaben der Standgerichte, Tagesberichte der Einsatzgruppe VI der Sicherheitspolizei und des SD, Dienstanweisungen, Richtlinien für den Verwaltungsaufbau.

Lit.: S. NAWROCKI: Poznan w okresie zarzadu wojskowego (Wrzesien - pazdziernik 1939 r.). 1972.

\section{Chef der Zivilverwaltung beim Oberbefehlshaber der 8. Armee im Militärbezirk} Lodsch

LO, Best. 9

Der Bestand (41 Bde, 1939) enthält Tages- und Stabsbefehle, einzelne Akten der Verwaltungsabteilung, u. a. über die Deutschen in der Wojewodschaft Lodz und Teilen der Wojewodschaft Posen, über Organisation der Zivilverwaltung in den Wojewodschaften Lodz, Warschau, Kielce, über Lager für Polen, Maßnahmen gegen polnische Intelligenz, Austausch von Kriegsgefangenen und Zivilflüchtlingen zwischen dem Deutschen Reich und der Sowjetunion (2 Bde, 1939-1940), Beiträge zu Tagesmeldungen, ferner aus der Kirchen- und Schul- (2 Bde), der Kriegswirtschaftlichen (14 Bde, dabei Unterlagen über die Lage der Industrie in Lodz, Beschäftigungslage und Preise, Lageberichte für Oktober 1939) und der Verkehrsabteilung (3 Bde), außerdem Aktensplitter der Außenstellen Radom und Warschau (2 Bde).

Verfilmt wurden (BA P, Best. PL-121, 2 Filme von 19 Bden) das Verordnungsblatt für den Militärbezirk, Schriftwechsel über Personalangelegenheiten mit Fragebögen aller Angestellten und Arbeiter, Tages- und Stabsbefehle, u. a. über Festnahmeaktionen, Aufbau von NSDAP und Polizei, Rückführung von Flüchtlingen und Zivilgefangenen, Einrichtung des Sondergerichts, Berichte der Außenstelle Warschau.

\subsection{Militärische Dienststellen mit regionaler Zuständigkeit und festen Standorten}

\subsubsection{Heeres- und Wehrmachtdienststellen}

\subsubsection{Stellvertretende Generalkommandos und Befehlshaber in den Wehrkreisen}

Die Ziffern in den folgenden Angaben zu Beständen des BA-MA bezeichnen die Stabsabteilungen (vgl. Teil I, S. 441). 
Wehrkreis I, Königsberg

BA-MA, Best. RH 53-1

Stammtafeln (18 Bde, auch für die Kriegsgefangenen-Stalags I A, B und F), Aktenreste (11 Bde, 1920-1945), dabei Unterlagen zur Feldparade 1937 und KTB des Wehrkreisarztes (Januar-Februar 1945).

Wehrkreis II, Stettin

BA-MA, Best. RH 53-2

Stammtafeln (16 Bde, auch für die Kriegsgefangenen-Stalags II A - E und -Oflags II A - E), Aktenreste (10 Bde, 1920-1944), u. a. betr. Manöver (3 Bde, 1933-1938), weitere unverzeichnet in Stettin (Best. 311, 10 Bde, 1933-1944).

Wehrkreis III, Berlin

BA-MA, Best. RH 53-3

Stammtafeln (22 Bde, auch für die Kriegsgefangenen-Dachdecker- und -Glaserbataillone), Aktenreste des Chefs des Stabes (15 Bde, 1919-1945), u. a. über Manöver 1936 und Vorbereitung der Verteidigung Berlins 1945, des Ia (5 Bde, 1920-1942, dabei Geschäftsverteilungspläne 1941 und 1942), Ib (7 Bde, 1941-1945), Ic (4 Bde, 1937-1944), u. a. über Zusammenarbeit mit dem Landeskriegerverband Ost (1939), Versorgung von Volkssturmeinheiten (1945), und des Korpsrichters über Strafverfahren (Januar bis April 1945).

Wehrkreis IV, Dresden

BA-MA, Best. RH 53-4

Stammtafeln (20 Bde), Aktenreste des Ia (14 Bde, 1929-1945, vor allem über Ausbildung von Führergehilfen, mit Geschäftsverteilungsplänen 1939 und 1943), Ib (11 Bde, 1934, 1944-1945), Ic (2 Bde), IIa (2 Bde), Militärgeographischen Dienstes (9 Bde, 1937-1945); dabei auch Unterlagen über Einsatz und Behandlung von Kriegsgefangenen durch das Landesschützenbataillon 396 (1942-1944).

Ergänzungsüberlieferung enthält der Sammelbestand Militär- und Heeresbauämter Dresden (DD, $1 \mathrm{lfm}$ und 4136 Baupläne 1874-1948) mit u. a. dem Amtsblatt der Wehrkreisverwaltung (1942), Richtlinien für militärische Neubauten (1931-1936), Übernahme von Gebäuden (1935-1942), Übersicht über Bauvorhaben (1931-1940), Richtlinien für den Bau von Anlagen zur Nahkampfausbildung, Schießstände und Munitionslager (1938-1948), Telefonverzeichnissen (1940).

Wehrkreis VIII, Breslau

MAW

Aktenreste (37 Bde, 1934-1943) betreffen die Garnison Breslau 1939-1942) und andere Standorte im Wehrkreis.

Wehrkreis XVII, Wien

BA-MA, Best. RH 53-17; AdR, Best. 08R300/2 Stammtafeln (16 Bde), Aktenreste des Ia (6 Bde, 1939-1944), in größerem Umfang des Ib (65 Bde, 1938-1945), u. a. betr. Organisation mit Unterlagen über RADEinsatz, Ersatzgestellung für Führerbegleitpersonal, Landesschützeneinheiten im Protektorat Böhmen-Mähren, innere Sicherheit, fremdvölkische Verbände des Heeres und der Waffen-SS, Verwendung italienischer und ungarischer Soldaten 1944/45, MobVorbereitungen 1938/39 mit Dienstanweisungen für Kommandanten von Kriegsgefangenen-Stalags und -Dulags, des Militärgeographischen Dienstes (6 Bde, 1938-1944), des IIa (8 Bde, 1938-1944, dabei Stabsbefehle) und des Kommandeurs der Kriegsgefangenen (11 Bde, 1941-1944) mit Lagerordnung für das Stalag XVII in Kaisersteinbruch, Meldungen über geflohene Gefangene und Bestrafungen, Unterlagen über italienische Militärinternierte. 
Der Wiener Bestand wird durch Aussonderung nach 1938 entstandener Akten aus der im früheren Kriegsarchiv vereinigten Gesamtüberlieferung des Bundesheeres gebildet und wird auch Schriftgut des Wehrkreises XVIII und der aus dem Bundesheer hervorgegangenen Divisionen und anderer Stäbe und Dienststellen der Wehrmacht in Österreich aufnehmen. Einen besonderen Bestand (07R100/1) bilden die Akten des ab 1939 dem Wehrkreiskommando (IIa) unterstellten früheren Archivstabs des Ministeriums für Landesverteidigung (54 Kts, überwiegend 1938-1940), die vor allem Wiedergutmachungsanträge vor 1938 aus politischen Gründen entlassener Angehöriger des Bundesheeres und Unterlagen über die Wiederaufnahme von Disziplinarverfahren enthalten.

Wehrkreis XVIII, Salzburg

BA-MA, Best. RH 53-18 Stammtafeln (16 Bde), Aktenreste des Ia (5 Bde, 1938-1939), Ib (8 Bde, 1938, 19431945) und des Militärgeographischen Dienstes (16 Bde, 1936-1944), Tätigkeitsbericht des Verbindungsoffiziers beim Höheren SS- und Polizeiführer Alpenland über Partisanen in Oberkrain und Untersteiermark (März 1943), KTB des GrenzwachtAbschnittkommandos XVIII G (Juli-Dezember 1944), vor allem jedoch Berichte, Bilder und anderes Material der Abteilung Propaganda (327 Bde) über einzelne Waffengattungen, auch Landesschützeneinheiten, Ausstellungen, Paraden, den Einmarsch 1938, die Besetzung des Sudetenlandes, den Einsatz von Einheiten des XVIII. Armeekorps bei den verschiedenen Feldzügen, einzelne Offiziere, vormilitärische Ausbildung durch die HJ.

Lit.: O. TUIDER: Die Wehrkreise XVII und XVIII, 1938-1945. 1975.

Wehrkreis XX, Danzig

BA-MA, Best. RH 53-20 Stammtafeln (8 Bde), Aktenreste des Ia (21 Bde) mit KTB des Militärbefehlshabers Danzig-Westpreußen mit Anlagen (September 1939-Dezember 1940), Tagebüchern und Tätigkeitsberichten (1942-1943), KTB des IV c (1939), Ic-Berichte über die politische und militärische Lage (2 Bde, 1939-1941).

Wehrkreis XXI, Posen

BA-MA, Best. RH 53-21

Stammtafeln (9 Bde), KTB des Militärbefehlshabers Posen mit Anlagen (4 Bde, 1939), des Ia (7 Bde, 14. Januar-5. Februar 1945, mit Sonderbeilage über Einsatz des Volkssturms), Aktenreste des Ic und der Verwaltung (je $1 \mathrm{Bd}$ ). Über einen Teilbestand in Warschau (MAW, 133 Bde, 1942-1945) sind keine Angaben möglich.

\subsubsection{Rüstungskommandos und -inspektionen}

Von den Rüstungsinspektionen in den Wehrkreisen sind in der Regel die Kriegstagebücher, monatliche Lageberichte und eine Zusammenfassung als „Geschichte der Rüstungsinspektion“ (1939-1944), von den nachgeordneten Rüstungskommandos die Kriegstagebücher (1939-1944) überliefert; auf zusätzliche Überlieferung wird jeweils hingewiesen.

Rüstungsinspektion I (Königsberg, 22 Bde).

Rüstungsinspektion II (Stettin, 13 Bde, außerdem Denkschrift über die wehrwirtschaftliche Lage im Bezirk 1938) und Rüstungskommandos Schwerin und Stettin (je 2 Bde, 1943-1944). 
Rüstungsinspektion III (Berlin, 18 Bde) und Rüstungskommandos Frankfurt/Oder ( 9 Bde, 1940-1944, und Denkschrift von 1939) und Potsdam (7 Bde, 1940-1944).

Rüstungsinspektion IV (Dresden, 20 Bde) und Rüstungskommandos Chemnitz (20 Bde), Dessau (7 Bde, 1942-1944, außerdem Listen von Personal der JunkersFlugzeugwerke 1944), Dresden (19 Bde), Halle (19 Bde) und Leipzig (20 Bde).

Rüstungsinspektion IVb (Reichenberg, 6 Bde, 1943-1944) und Rüstungskommando Reichenberg (5 Bde, 1939-1943).

Rüstungsinspektion VIII (Breslau, 28 Bde, außerdem Denkschriften über Wehrwirtschaft in Niederschlesien um 1937 und Wanderbewegung 1938, KTB des Wehrwirtschaftsstabs z.b.V. 1939-1940) und Rüstungskommandos Breslau (9 Bde, 1942-1944) und Liegnitz (11 Bde).

Rüstungsinspektion VIIIb (Kattowitz, 6 Bde, 1943-1944) und Rüstungskommandos Gleiwitz (5 Bde, 1943-1944), Kattowitz (4 Bde, 1939-1943) und Troppau (8 Bde) mit Ergänzungsüberlieferung des Oberschlesischen Instituts für Wirtschaftsforschung (KAT, 42 Bde, vor allem Gutachten und Denkschriften, 1942-1945).

Rüstungsinspektion XVII (Wien, 15 Bde) mit Rüstungskommandos Linz (13 Bde), Wien (6 Bde) und Wien-Mödling (14 Bde).

Rüstungsinspektion XVIII (Salzburg, 28 Bde) mit Rüstungskommandos Graz (22 Bde), Innsbruck (18 Bde) und Klagenfurt (14 Bde, 1941-1944).

Rüstungsinspektion XX (Zoppot, 14 Bde).

Rüstungsinspektion XXI (Posen, 15 Bde) mit Rüstungskommandos Litzmannstadt (6 Bde) und Posen (6 Bde, 1942-1944, mit Liste der Wehrmachtbetriebe).

Außerdem ist Schriftgut der Rüstungskommandos Eisenach (17 Bde) und Weimar (12 Bde) sowie Magdeburg (8 Bde, 1942-1944) vorhanden, die zu den westdeutschen Wehrkreisen IX bzw. XI gehörten.

Lit.: GUIDES to German Records, vol. 7 und 17. - K. M. POSPIESZALSKI: Memorial pt. „Die Bedeutung des Polen-Problems für die Rüstungswirtschaft Oberschlesiens“. 1945. - A. SZEFER: „Kriegstagebuch“ jako zrodlo do dziejow regencji katowieckiej w latach okupacjii hitlerowskiej. 1971.

\subsubsection{Wehrersatzinspektionen, Wehrbezirkskommandos und Wehrmeldeämter}

Von den bei den Wehrbezirkskommandos in Österreich geführten Wehrstammbüchern und ergänzenden Unterlagen aus Wehrmeldeämtern blieb ein relativ großer Teil erhalten (AdR, Best. 08R001, ca. 500000 Stück, dazu im Best. O8R004 ca. 120000 Karteikarten verschiedener Provenienzen und im Best. 08R050 Wehrstammrollen insbesondere aus Kärnten, auch von Wehrpflichtigen aus Südmähren und der Untersteiermark); sie stammen vor allem von den Wehrbezirkskommandos bzw. Wehrmeldeämtern St. Pölten, Baden, Wiener Neustadt, Judenburg, Leibnitz, Leoben. Aktensplitter der Wehrersatzinspektion und des Wehrbezirkskommandos Innsbruck (4 bzw. 3 Bde 
Befehlssammlung 1943-1945) und des Wehrbezirkskommandos und Wehrmeldeamtes Salzburg (25 Bde, 1938-1945, u. a. Stabsbefehle, Unterlagen über ausländische Freiwillige) gelangten ins BA-MA (Best. RW 15).

Sehr viel dürftiger ist die Überlieferung aus Mittel- und Ostdeutschland und den eingegliederten Ostgebieten. Sie beschränkt sich auf Restakten des Wehrbezirkskommandos Gleiwitz (KAT, Außenstelle Gleiwitz, 101 Bde, 1941-1945), Wehrpässe und ähnliche Unterlagen des Wehrbezirkskommandos Litzmannstadt (ZDM MO, Best. 1360, 727 Bde, überwiegend 1944), Wehrstammbücher des Wehrbezirkskommandos Schwerin (BA DH, künftig ZNS, 5 lfm, 1939-1944) und einzelne Aktensplitter verschiedener anderer Dienststellen (BA-MA und BA DH).

Lit:: O. WINTER: Personalunterlagen des Zweiten Weltkrieges im Kriegsarchiv. 1975. - G. ARTL: Die Bestandsgruppe „Deutsche Wehrmacht“ - Personenauskünfte im Dienste der Öffentlichkeit. 1992.

\subsubsection{Kriegsgefangenenlager}

Die relativ umfangreichste Überlieferung konnte im Staatsarchiv der RSFR in Moskau (Best. 7021-105, 41 Bde, 1942-1945, Kopien daraus im Staatsarchiv Detmold) ermittelt werden; sie stammt aus dem Stalag 326 VI K im westfälischen Stukenbrock. Die Akten enthalten u. a. allgemeine Dienstvorschriften und Befehle und betreffen vor allem Kriegsgefangene aus der Sowjetunion, deren Behandlung und Bestrafung von Fluchtversuchen, Sterblichkeit. Aktenreste sind aus dem Stalag III A in Luckenwalde vorhanden (BA-MA, Best. RH 49, 11 Bde; ZDM MO, Best. 1367, 12 Bde, vor allem über französische Kriegsgefangene). Lediglich Aktensplitter gibt es (BA-MA, Best. RH 49, soweit nichts anderes vermerkt) von den Stalags I A, II A (Neubrandenburg), II D (Stargard), IV A (Hohnstein, dazu Bilder des Landesschützenbataillons 393 BA DH), IV B, IV D, IV E (Altenburg), IV G (Oschatz), VIII C (Lamsdorf), IX E (Bad Sulza, 3 Bde, 1941-1945, ZDM MO, Kommandanturbefehle 1944-1945 BA DH), XVII B (Gneixendorf, Totenbuch 1943-1945 AdR, in Best. 08R700) und den Oflags IV C (Torgau, Colditz) und XVII (Edelbach). Karteikarten über polnische Kriegsgefangene jüdischer Abstammung, die in das $\mathrm{KL}$ Lublin überführt und dort ermordet wurden, gelangten an das Jüdische historische Institut in Warschau. Listen in Luckenwalde, Belsen, Sandbostel, Bitterfeld und anderen Lagern 1942 bis 1944 verstorbener Kriegsgefangener (ZDM MO) stammen wahrscheinlich von der Wehrmachtauskunftstelle.

Lit:: V. SCHOCKENHOFF: Neue Quellen zur Geschichte der sowjetischen Kriegsgefangenen im Deutschen Reich. 1993. - S. LAGODZINSKI: Stalag I B Hohenstein: W swietle zeznan swiadkow 1977. - A. ZIENTARSKI: Oboz jeniecki w Czarnem. Stalag II B Hammerstein. 1975. - J. BOHATKIEWICZ: Oflag II B Arnswalde. 1974. - M. SADZEWICZ: Oflag II D Groß-Born. 1977. - S. SENFT: Rozmieszczenie, liczebnosc i sklad narodowosciowy obozow jenieckich w VIII okregu wojskowym Wehrmachtu podczas II wojny swiatowej. 1974. - A. PLUCINSKI: Fragmenty notatek prowadzonych w Stalagu VIII B Lamsdorf w okresie od 29 padziernika 1944 do 20 stycznia 1945 r. 1978. - T. SOJKA: Odnalzienie zweglonych dokumentow z archiwum Stalagu VIII C w ZaganiuJ. 1975. - L. URYGA: Stalag VIII B. 1978. - ders.: Stalag 318 (VIII F). 1974. - J. TYSZEWICZ: Stalag XX A, oboz jencow wojennych w Toruniu w latach 1939-1945. 1976.

\subsubsection{Abwehrstellen}

Abgesehen von Aktensplittern, darunter Rundverfügungen, der Abwehrstelle IV, Dresden (BA DH, 4 Bde, 1936-1940), sind nur Akten der Abwehrstelle im Wehrkreis II, Stettin, überliefert (ZDM MO, Best. 1147, 97 Bde, vielfach wenig umfangreich, 19351944), vermutlich im Zusammenhang mit der Übertragung von Zuständigkeiten auf die Staatspolizeileitstelle Stettin. Sie betreffen u. a. Geschäftsverteilung und Organisation 
(15 Bde), Untersuchung von Sabotagefällen (4 Bde), Erhebungen zur Wirtschaftslage (3 Bde, 1936-1940), die Auswertung erbeuteter Akten des polnischen Nachrichtendienstes, Lieferung erbeuteter polnischer Flugzeuge an Bulgarien, hauptsächlich aber die Überprüfung der Sicherheitsmaßnahmen in Rüstungsbetrieben, ihrer Mitarbeiter und die Bestellung von Abwehrbeauftragten.

\subsubsection{Geheime Feldpolizei Bad Nauheim ZDM MO, Best. 1369}

Das Kriegstagebuch nebst Anlagen (4 Bde, Januar-November 1940) und einige Akten geben Aufschluß über Organisation und Personalangelegenheiten (9 Bde, 1939-1941) und die Zusammenarbeit mit anderen Polizeidienststellen (13 Bde) bei Sicherungsaufgaben im Taunus (Usingen, Reifenberg) beim Aufmarsch für den Frankreichfeldzug sowie den späteren Einsatz in Frankreich, Griechenland und der Sowjetunion (4 Bde).

\subsubsection{Divisionskommandos vor 1939}

Während über den Inhalt des Schriftguts der 2. Infanteriedivision in Stettin (MAW, 323 Bde, 1926-1940) keine Angaben möglich sind, läßt sich sagen, daß von der 32. Infanteriedivision in Köslin (MA W, 630 Bde, 1933-1944) u. a. folgende Akten vorhanden sind:

Befehle u. a. über den Angriff auf Polen 1939, Kriegstagebuch, Mob-Pläne, Berichte, Schulungs- und Manöverprogramme, Unterlagen über die organisatorische Gliederung des Stabes (1939-1942), Propaganda, Bestrafungen, Befehle der Kriegsmarine (1940-1941), Übersichten über die wehrwirtschaftliche Lage und über die Streitkräfte Frankreichs, Polens, der Tschechoslowakei, Litauens, der Sowjetunion und Japans.

\subsubsection{Truppenteile vor $1939 \quad$ BA-MA, Best. RH 37}

Vorhanden sind vor allem Akten des Infanterieregiments Nr. 72 mit den Standorten Karlsbad, Eger und Pilsen (ca. 100 Bde, überwiegend 1938-1940); sie betreffen u. a. die Besetzung von Böhmen 1939 und das Verhältnis zur tschechischen Bevölkerung. Vom Infanterieregiment Nr. 101, das in Leisnig, Döbeln und Oschatz stationiert war, liegen nur Aktenreste vor (ca. 30 Bde, meist 1934-1940).

\subsubsection{Luftwaffe}

\subsubsection{Luftgaukommandos}

Von den für Mittel- und Ostdeutschland zuständigen Luftgaukommandos sind folgende Bestände (BA-MA, Best. RL 19) überliefert:

Luftgaukommando I, Königsberg: Unterlagen u. a. über Einsatz der Flak 1943, Angriffe auf Königsberg und Gotenhafen 1943, Verfahren vor dem Feldgericht, Besichtigung der Kriegsgefangenenlager Albertshof und Heydekrug (5 Bde, 1942-1944).

Luftgaukommando II, Stettin: Überwiegend Akten der Quartiermeister-Abteilung und über Organisation, u. a. über Einsatz der NSKK-Brigade Nr. 2 (9 Bde, 1939-1941).

Luftgaukommando III, Berlin: KTB des Ia 1940 mit Angaben über Luftangriffe auf Berlin, Brandenburg, Jüterbog, Magdeburg und Stettin, Akten über Verwaltungsangelegenheiten unterstellter Einheiten (9 Bde, 1940-1945). 
Luftgaukommando IV, Dresden: Aktensplitter (5 Bde, 1939-1941).

Luftgaukommando VIII, Breslau: Überwiegend Verwaltungsakten, insbesondere über Erwerb und Verwaltung von Liegenschaften, auch für Wohnungen (95 Bde, 1937-1945, mit Listen von Rüstungsbetrieben auf Anlagen der Luftwaffe 1944-1945), ferner Tätigkeitsberichte und Organisationsunterlagen mit KTB-Anlagen (8 Bde, 1942-1944) mit Angaben über Rechtspflege und Kriegsgefangene, Akten über Personalverwaltung (27 Bde, 1937-1944) und Dienstbetrieb (12 Bde, 1936-1944) mit Unterlagen über Luftwaffenhelfer, Fremdarbeiter und jüdische Zwangsarbeiter (1943). Aus der Außenstelle Kattowitz sind Befehle und Anweisungen mit Informationen über die Organisation der Luftverteidigung in Schlesien, Dislozierung der unterstellten Einheiten, Taktik der Bekämpfung der feindlichen Verbände und zivilen Luftschutz vorhanden (MA W, 24 Bde, 1943-1945).

Aus dem für Österreich mit Ausnahme von Tirol-Vorarlberg zuständigen Luftgaukommando XVII, Wien, liegen (AdR, Best. 08R401/2, 26 Kts, überwiegend 19381940) vor allem Akten über Personalangelegenheiten von Offizieren (Einzelpersonalakten, -karteikarten, auch von Luftwaffenhelfern und -helferinnen, und Gebührnisunterlagen von Zivilbediensteten in den Beständen 08R001 und -02) mit Bewerbungen, Beurteilungen, Vorgängen über Entlassungen, Übernahme von Polizeioffizieren, Lehrgänge vor, außerdem eine unvollständige Reihe von Tagesbefehlen (1939-1940), ein Geschäftverteilungsplan des Stabes, Merkblätter und Verordnungen, Abendmeldungen über Luftangriffe in Akten der Gauleitung Wien (1942-1945, in Best. 08R450). Ein Teilbestand (BA-MA) enthält neben einer Anlage zum KTB des Ic (August 1943) Akten über einzelne Liegenschaften der Luftwaffe im Protektorat Böhmen und Mähren (110 Bde, 1939-1944).

\subsubsection{Verbände der Jagdflieger im Reichsgebiet BA-MA, Best. RL 10} Lediglich aus dem Einsatz je einer Gruppe des Jagdgeschwaders 102 in Ostpreußen 1944, des Nachtjagdgeschwaders 5 in den Räumen Kolberg, Stendal und Parchim 1944-1945 und des Zerstörergeschwaders 26 in Königsberg/Neumark 1944 sind Unterlagen vorhanden.

\subsubsection{Flughafenbereichskommandos BA-MA, Best. RL 20}

Einzelne Akten stammen vor allem von den Kommandos Breslau, Döberitz, Jesau und Stendal, außerdem aus Altenburg, Friedeberg (Österreich), Heiligenbeil, Liegnitz, Merseburg, Neubrandenburg, Parchim, Posen und Schwerin.

\subsubsection{Einheiten der Flakartillerie im Reichsgebiet}

BA-MA, Best. RL 12

Aktenreste und Kriegstagebücher sind überliefert von den Flakregimentern 52 (Magdeburg, 1941), 90 (Leipzig, 1942-1945), 120 (Böhlen, Zeitz, betr. Einsatz von Frauen 19441945), 300 (Zeitz, Leipzig, Bitterfeld, 8 Bde, 1941-1945) und I./2 (Stettin, 1933-1937), von den schweren Abteilungen 132 (Halle, Merseburg, 1944-1945), 357 (Böhlen, 19441945) und 662 (Zeitz-West), der Reserve-Abteilung 522 (Magdeburg, KTB 1939-1940) und der Scheinwerferabteilung 370 (Raum Berlin, KTB 1944) sowie den Sperrfeuerbatterien 34, 35, 36, 39, 40 und 63, die in Ost- und Westpreußen (Elbing, Danzig, Königsberg, Graudenz) eingesetzt waren (KTBs 1940-1943). 
8.3.2.5 Luftschutzabteilungen BA-MA, Best. RL 13 Vorhanden ist nur das Kriegstagebuch der nach Angriffen auf Leipzig, Magdeburg und Köthen eingesetzten Luftschutzabteilung (mot) 48 (2 Bde, 1943-1944). 
